\title{
Die koloniale Gegenwart und dekoloniale Zukunft transnationaler Entwicklungspolitik
}

\author{
Prof. Dr. Daniel Bendix \\ Friedensau Adventist University \\ Daniel.Bendix@thh-friedensau.de
}

\section{Zusammenfassung}

Die Entwicklungspolitik der BRD ist ein transnationales Unterfangen, ein Zusammenspiel aus Aktivitäten im In- und Ausland. Dabei kann sie sowohl zur Stabilisierung als auch zur Destabilisierung kolonialer Kontinuitäten beitragen. In diesem Beitrag wird empirisch der Frage nach einer kolonialen Gegenwart und dekolonialen Zukunft bundesdeutscher Entwicklungspolitik nachgegangen. Für den ersten Fall wird die Bearbeitung des Themas Bevölkerungsentwicklung untersucht und auf Forschung in Deutschland und Tansania zurückgegriffen. Um die Möglichkeiten einer dekolonialen Zukunft bundesdeutscher Interventionen auszuloten, wird sich dem Bereich der Agrarpolitik zugewendet. Es wird argumentiert, dass eine Aufmerksamkeit für transnationale Verbindungen des kolonialen Erbes vonnöten ist, um dem proklamierten Ziel von Gleichheit und Gerechtigkeit der Entwicklungspolitik näher zu kommen.

Schlagwörter: Entwicklungspolitik; transnational; dekolonial; Deutschland; Bevölkerungspolitik; Agrarpolitik

\begin{abstract}
Germany's development policy is a transnational endeavour, an interplay of activities at home and abroad. It can contribute to stabilising as well as destabilising colonial continuities. This paper empirically examines the question of a colonial present and a decolonial future of German development policy. For the first case, and drawing on research in Germany and Tanzania, it investigates how the issue of demographic development is dealt with. To explore the possibilities of a decolonial future of German interventions, the paper turns to the field of agrarian policy. It is argued that attention needs to be paid to the transnational connections of the colonial legacy in order to come closer to development policy's proclaimed aim of equality and justice.

Keywords: development policy; transnational; decolonial, Germany, population policy, agrarian policy
\end{abstract}

\section{Einleitung}

Bundesdeutsche Entwicklungspolitik in Bezug auf den Globalen Süden findet nicht nur im Süden statt, sondern auch im Inland. Zu nennen sind diesbezüglich beispielsweise die Öffentlichkeits- und Informationsarbeit von entwicklungspolitischen Organisationen und staatlichen Institutionen, aber auch entwicklungspolitische Bildung in schulischen und außerschulischen Kontexten. Der entwicklungspolitische Freiwilligendienst Weltwärts ist paradigmatisch für die Verknüpfung von bundesdeutschen Aktivitäten im In- und Ausland. Im Rahmen ihrer Vor- und Nachbereitung 
nehmen Weltwärts-Freiwillige an Seminaren zu Globalem Lernen bzw. Bildung für nachhaltige Entwicklung teil; während des Auslandaufenthalt in einem sogenannten Entwicklungsland ,engagieren [sie] sich in einem Entwicklungsprojekt und nehmen Erfahrungen mit, die sie ihr ganzes Leben lang begleiten“ (BMZ 2019b); und laut BMZ ,engagieren sich die Rückkehrerinnen und Rückkehrer [nach ihrem Auslandsaufenthalt, DB] weiter in der entwicklungspolitischen Arbeit“ und „tragen [...] ihre Erfahrungen in die Gesellschaft und leisten über ihren Auslandseinsatz hinaus einen persönlichen Beitrag für eine gerechtere Welt“ (2019a).

In diesem Beitrag beschäftige ich mich mit dem transnationalen Zusammenwirken von bundesdeutscher Entwicklungspolitik im In- und Ausland, mit deren Wechselwirkung und auch Widersprüchlichkeit. Unter Entwicklungspolitik verstehe ich staatliche und nicht-staatliche Anstrengungen - von Initiativen, Einzelpersonen und privatwirtschaftlichen Akteuren -, Veränderungen im Globalen Süden bzw. im Nord-Süd-Verhältnis zu bewirken. Deutschland spielt heute entwicklungspolitisch, aber auch weltwirtschaftlich und geopolitisch eine wichtige Rolle im globalen Gefüge: Die BRD ist die drittgrößte Exportnation, dominiert die Wirtschaftspolitik der Europäischen Union und ist zentral daran beteiligt, die Grenzen der EU gegen Migrant*innen zu schließen. Zudem ist die BRD der weltweit zweitgrößte „Geber“von „Entwicklungshilfe“.

Aufbauend auf Arbeiten, die bundesdeutsche Entwicklungspolitik in ihrer (Dis-) Kontinuität zum Kolonialismus betrachten - konkret in Bezug auf Weltwärts (Haas 2012; Kontzi 2015), aber auch allgemeiner im Bereich Bildung (Bechtum/Overwien 2017; Danielzik 2013) oder Öffentlichkeitsarbeit (Kiesel/Bendix 2010) sowie in ihrer Wirkung im Süden (Bendix 2016) - verfolge ich in einem ersten Schritt die Frage nach den kolonialen Verwicklungen transnationaler Entwicklungspolitik. Es geht dabei, wenn wir uns mit der BRD beschäftigen, um den Umgang mit einer 500-jährigen Kolonialgeschichte, welche die BRD mit den heute als Entwicklungs- und Schwellenländer bezeichneten Gesellschaften verbindet (Friedrichsmeyer/Lennox/Zantop 1998). Die Stärke post- bzw. dekolonialer Kritik besteht darin, sich auf das Zusammenspiel von Wissen und Macht zu konzentrieren. Es wird entsprechend den Nachwirkungen von Kolonialismus „als ein Herrschafts-, Macht- und Ausbeutungssystem und [...] als ein Wissens- und Repräsentationssystem" nachgegangen (Hall 1996: 254). Dabei geht es post- bzw. dekolonialer Kritik in politischer Stoßrichtung immer um die Infragestellung kolonialer Dominanz und um eine Befreiung vom Erbe des Kolonialismus. So erörtere ich in einem zweiten Schritt die Möglichkeiten für eine weniger koloniale oder sogar dekoloniale Entwicklungspolitik.

Für den Aspekt der Analyse kolonialer Gegenwart ziehe ich die bundesdeutsche transnationale Bevölkerungspolitik heran. Grundlage sind Interviews mit deutschen Entwicklungsexpert*innen in Tansania und der BRD aus den Jahren 2009 
und 2010 sowie der vom BMZ und der Kultusministerkonferenz herausgegebene Orientierungsrahmen für den Lernbereich Globale Entwicklung. Indem betrachtet wird, wie Fragen von Bevölkerungsentwicklung transnational verhandelt werden, wird das Fortwirken kolonialer Verhältnisse beispielhaft aufgezeigt. Der Frage nach der Möglichkeit einer Dekolonisierung von bundesdeutscher Entwicklungspolitik wird auf der Grundlage von teilnehmender Beobachtung in Mali und Deutschland zu Widerstand gegen Landgrabbing im Jahr 2018 sowie der Analyse entsprechender entwicklungspolitischer Spendenwerbung im Jahr 2017 nachgegangen. Der Bereich der Agrarpolitik ist besonders relevant für die Aussicht auf eine dekoloniale Zukunft, weil darin Fragen des Zugangs zu und Zugriffs auf Land verhandelt werden, welche sowohl im Globalen Norden als auch Globalen Süden immer umkämpfter werden.

\section{Die koloniale Gegenwart transnationaler deutscher Bevölkerungspolitik}

Der Anspruch, die kolonisierten Gesellschaften zu „entwickeln“, war immer Teil von Kolonialismus, wie auch die Teilnehmenden an der Berliner Afrika-Konferenz von 1884/85 festhielten: „Alle Mächte [...] verpflichten sich, die Erhaltung der eingeborenen Bevölkerung und die Verbesserung ihrer sittlichen und materiellen Lebenslage zu überwachen“ (Deutsches Reichsgesetzblatt 1885: 225). Das galt zum Beispiel auch für Fragen von Bevölkerungsentwicklung. Deutsche Kolonisatoren äußerten ab 1900 ihre Besorgnis über einen „Bevölkerungsrückgang“ in den Kolonien (Deutsche Gesellschaft für Eingebornenschutz 1914). Das Ziel, die Bevölkerungsgröße zu erhöhen, war vor allem von der Sicht auf die Kolonisierten als ökonomische Ressource getrieben. Sie sollten ausgebeutet werden, mussten dafür aber eben auch „erhalten“werden.

Einwirkungen auf Bevölkerungsentwicklung sind auch heute Teil deutscher Interventionen im Globalen Süden. Deren Betrachtung ist erhellend für ein Verständnis kolonialer Nachwirkungen in der Entwicklungspolitik. Die BRD ist entwicklungspolitisch im Bereich „reproduktive Gesundheit“ und „Bevölkerungsentwicklung" aktiv, insbesondere in Afrika (BMZ 2013). Die aus deutscher Sicht zu hohen Geburtenraten in vielen afrikanischen Ländern werden von deutschen Entwicklungsexpert*innen in Interviews als Zeichen für gesellschaftliche Rückständigkeit interpretiert. So äußert sich eine für den Bereich Gesundheit zuständige Mitarbeiterin der Kreditanstalt für Wiederaufbau (KfW) exemplarisch zu Tansania:

Man sieht eben auch, dass die Fertilitätsrate noch unverändert hoch ist, dass der gesamte Kontext in Tansania immer noch sehr konservativ ist, was Frauen anbelangt, [...] und damit eben auch eine gewisse Unterdrückung einhergeht. Und insofern ist noch ein großer Nachholbedarf gerade in diesem Bereich in Tansania (Interview mit Mitarbeiterin der KfW, 18.12.2010). 
Die deutsche Entwicklungszusammenarbeit (EZ) nimmt dabei an, dass der Grund für ihrer Ansicht nach zu hohen Geburtenraten unter anderem hoher ungedeckter Bedarf an Verhütungsmitteln ist. Im konkreten von mir untersuchten Fall von deutscher Entwicklungspolitik in Tansania werden die „Entwicklungsexpert*innen“ damit konfrontiert, dass entgegen ihrer Vorannahme

erstaunlicherweise das Interesse oder die Nachfrage an Kontrazeptiva [...] gar nicht so hoch ist. [...] In Tansania ist die Herausforderung [...] was können wir als deutsche EZ [tun], um diese Nachfrage ... also das Ministerium und die Zivilgesellschaft dabei zu unterstützen, diese Nachfrage zu generieren (Interview mit Leiter*in der Komponente Reproduktive Gesundheit des Tanzanian-German Programme to Support Health, 19.03.2010).

Erst jüngst hat auch die Stiftung Wissenschaft und Politik, der wichtigste deutsche Thinktank für Außen- und Sicherheitspolitik, festgehalten, dass nicht unbedingt ein ungedeckter Bedarf an Verhütungsmitteln, sondern ein zu hoher Kinderwunsch in vielen afrikanischen Ländern als Problem angegangen werden sollte (Angenendt/ Popp 2014: 27). Während mit Verweis auf die Menschenrechte „freie Wahl“ der Kinderzahl propagiert wird, werden Afrikaner*innen als irrational bewertet, wenn sie nicht weniger Kinder haben oder „moderne“Verhütungsmittel verwenden wollen.

Diese kolonial-paternalistischen Diskurse sind von handfesten ökonomischen Interessen begleitet (Bendix/Schultz 2017). Das Pharmaunternehmen Bayer hat beispielsweise zusammen mit USAID die „Contraceptive Security Initiative“ ins Leben gerufen, um Verhütungspillen in Tansania und in weiteren zehn afrikanischen Ländern für wohlhabendere Bevölkerungsschichten im privaten Markt zu etablieren. Die „Contraceptive Security Initiative“ stellt nach Bayer (2011) „einen neuen strategischen Ansatz und einen innovativen Weg zur Erschließung der Märkte in Entwicklungsländern dar“. 2012 gründete Bayer darüber hinaus zusammen unter anderem mit der britischen und US-amerikanischen Regierung das „Jadelle Access Program“. Bayer und die Bill \& Melinda Gates Foundation vereinbarten, 27 Millionen Stück des Verhütungsimplantats Jadelle über sechs Jahre hinweg für einen reduzierten Preis für entwicklungspolitische Maßnahmen zur Verfügung zu stellen und verlängerten die Zusammenarbeit kürzlich. Der globale Kontrazeptivamarkt soll sich bis 2020 auf 19,6 Milliarden US-Dollar steigern, was einer Wachstumsrate von jährlich 3,1 Prozent entspricht (Transparency Market Research 2014). Die BUKO Pharma-Kampagne wertet die „Contraceptive Security Initiative“ entsprechend als „Marketinginstrument, um Bayers Position als Weltmarktführer für orale Kontrazeptiva und auch die Marktstellung im Bereich Frauengesundheit zu verteidigen“ (2014: 40).

Zusammenfassend kann gesagt werden, dass heute wie zur Kolonialzeit unterschiedliche deutsche Akteure es als ihre Aufgabe ansehen, die Bevölkerungsentwicklung in Afrika zu beeinflussen, wobei der koloniale Blick mit ökonomischen 
Interessen verquickt ist. Aber die Vorstellung, dass wir uns in Deutschland für Fertilitätsraten und Bevölkerungsentwicklung im Süden interessieren sollten, ist nicht selbstverständlich. So ist es beispielsweise andersherum nicht der Fall - Tansania schickt keine Expert*innen nach Deutschland, um unser Reproduktionsverhalten zu verändern. Der unidirektionale „Entwicklungsblick“ (Escobar 1994: 155) muss im Inland immer wieder geschaffen werden.

Hier ist beispielsweise die Deutsche Stiftung Weltbevölkerung (DSW) bedeutsam. Sie versucht, die deutsche Gesellschaft von dem Problem einer wachsenden Weltbevölkerung zu überzeugen. Die DSW machte 2011 zum „Tag der Sieben Milliarden" katastrophistische Aussagen wie die, dass die Zahl der Menschen auf der Erde um 2,6 pro Sekunde, 158 pro Minute, über 225.000 pro Tag oder 83 Millionen pro Jahr wächst (Deutsche Welle 2012). Sie hat auch zwei entsprechende „Weltbevölkerungsuhren“ in Hannover aufgestellt, eine davon ausgerechnet vor dem Zoo.

Aber auch in der schulischen und außerschulischen entwicklungspolitischen Bildungsarbeit geht es um das Thema Bevölkerungsentwicklung. Im „Orientierungsrahmen für den Lernbereich Globale Entwicklung“ (KMK/BMZ 2007: 2015) ist einer der zentralen Themenbereiche „demographische Strukturen und Entwicklungen“. Im Teil zum Fach Geschichte wird die Bevölkerungsfrage in den Zusammenhang mit globalen Umweltveränderungen gestellt und vorgeschlagen, „Bedingungen und Folgen des Wachstums der Weltbevölkerung in Vergangenheit und Gegenwart“ zu behandeln (KMK/BMZ 2015: 248). Es wird dabei immer wieder suggeriert, dass die Anzahl der Menschen ein Problem für „Entwicklung“ sei, nicht die Art und Weise, wie der Zugriff auf Ressourcen und Natur durch Kapitalismus und koloniale Ausbeutungsstrukturen organisiert ist (Brand/Wissen 2017).

In dem öffentlichen Entwurf des Orientierungsrahmens war das Unterrichtsbeispiel „globale Bevölkerungsentwicklung“ für das Fach Mathematik vorgeschlagen worden. Das Thema wurde dabei folgendermaßen eingeführt:

Die unterschiedliche Entwicklung der Weltbevölkerung, ihre schnelle Zunahme in Teilen der Welt aber auch ihre Abnahme und die Alterszunahme in einigen Ländern, stellen eine ernst zu nehmende Herausforderung dar. Die Versorgung mit Nahrung, Trinkwasser, Energie und elementaren Konsumgütern gelingt trotz erheblicher Fortschritte nicht hinreichend. Zudem geht eine Anhebung des Lebensstandards oft direkt oder indirekt zu Lasten der natürlichen Umwelt und damit der Lebensgrundlagen (KMK/BMZ 2014: 235).

Während der erste Satz zur Bevölkerungsentwicklung insgesamt in alarmistischer Manier als „ernst zu nehmende Herausforderung“ bezeichnet wird, zeigt der zweite Satz, wohin die Reise geht: Der Blick der Schüler*innen soll in den Süden wandern, denn nur dessen Bevölkerung kann mit Defiziten in der Versorgung mit „Nahrung, Trinkwasser, Energie und elementaren Konsumgütern“ in Verbindung 
gebracht werden. Im dritten Satz geht es um das Thema übermäßiger Konsum, aber es bleibt ungeklärt, wie der mit „Abnahme der Bevölkerung und Alterszunahme in einigen Ländern" und damit mit dem Globalen Norden in Verbindung stehen soll. Insofern kann auch dies nur wieder als Problem von sogenannten Entwicklungs- und Schwellenländern betrachtet werden. Dabei wird nicht reflektiert, wie reduktionistisch eine Perspektive ist, die Bevölkerungsfragen rein quantitativ diskutiert; und dabei bevölkerungspolitische Ideologien und Ungleichheit innerhalb eines globalen, rassialisierten Kapitalismus nicht zum Thema macht. Eine Perspektive auf Entwicklung, die politische, soziale und ökologische Probleme zu demographischen Fragen macht, durchzieht den gesamten Orientierungsrahmen. Anzumerken ist, dass das Unterrichtsbeispiel für das Fach Mathematik unter anderem nach Kritik einer Initiative, die sich „decolonize Orientierungsrahmen!“ (2014) nennt und an der ich mit dem Verein glokal beteiligt war, in der Endfassung von 2015 ersatzlos gestrichen wurde (KMK/BMZ 2015).

\section{Die dekoloniale Zukunft deutscher Agrarpolitik}

Nachdem ich am Beispiel von bevölkerungspolitischen Themen aufgezeigt habe, wie sich eine in kolonialer Kontinuität stehende Entwicklungspolitik nach außen und innen transnational ergänzt und stabilisiert, widme ich mich nun der Frage, inwiefern in den Aktivitäten bundesdeutscher Akteure zur Frage des Zugangs zu Land eine dekoloniale Zukunft durchschimmert. In zahlreichen konkreten Fällen wie auch in bundesdeutschen entwicklungspolitischen Agrarinitiativen wie dem German Food Partnership oder dem Africa Agriculture and Trade Investment Fund wird deutlich, dass die deutsche staatliche Entwicklungspolitik den Interessen von Investor*innen größere Bedeutung zumisst als den von Kleinbäuerinnen und -bauern (Brämer/Ziai 2015). Ein bekanntes Beispiel ist, dass die Bundesregierung die Hamburger Neumann Gruppe zum Nachteil der von der ugandischen Kaweri Kaffeeplantage vertriebenen Landbevölkerung unterstützt (FIAN 2017). Der ehemalige Minister des BMZ, Dirk Niebel, stellte klar: „Die Kaweri Plantage ist die größte deutsche Investition in Uganda und hat [...] das Wohlwollen der deutschen Regierung" (Lincoln 2013). Die agroindustrielle Produktion bevorzugende Ausrichtung der Bundesregierung wurde im Vorlauf zum G20-Gipfel in Hamburg mit dem „Compact with Africa“ des Finanzministeriums sowie dem „Marshallplan mit Afrika“ des BMZ erneut deutlich (Bernau 2017).

Wie wird die Frage von Zugang zu Land nun entwicklungspolitisch der deutschen Öffentlichkeit vermittelt? Üblicherweise werden Menschen aus dem Globalen Süden in der Öffentlichkeitsarbeit und insbesondere der Spendenwerbung von Hilfsorganisationen in kolonialer Weise als hilfsbedürftig und abhängig dargestellt (Kiesel/Bendix 2010). Besonders nach der Krise des Kapitalismus und der damit zusammenhängenden Nahrungsmittelkrise 2007/2008 war allerdings eine 
kurzzeitige Politisierung der Öffentlichkeitsarbeit von entwicklungspolitischen Organisationen zu sehen. So wurde beispielsweise davor gewarnt, mit Lebensmitteln zu spekulieren, womit unsere Lebensweise in direktem Zusammenhang mit der Landwirtschaft und damit der Lebensmittelversorgung von Menschen im Süden gestellt wird (Brot für die Welt 2013a; 2013b). Misereor (2011) startete eine großangelegte Plakataktion mit 100.000 Bildern über ganz Deutschland verteilt. In einem Plakat geht es auch um das Thema Landgrabbing. Hier wird zur Unterstützung von Kämpfen im Süden aufgefordert und ganz explizit die politische Handlungsfähigkeit von Menschen im Süden hervorgehoben.

Ich selbst beschäftige mich derzeit mit den Aktivitäten von Afrique-Europe-Interact (AEI), in denen ich eine dekoloniale Zukunft der Entwicklungspolitik aufscheinen sehe. Dieses transnationale Netzwerk setzt sich aus Basisaktivist*innen in Mali, Togo, Guinea, Burkina Faso, Marokko, Deutschland, Österreich und den Niederlanden zusammen. Es wird von Aktivist*innen aus Deutschland koordiniert. Politisch verfolgt AEI zwei Ziele: Einerseits wird die EU- und deutsche Migrationspolitik problematisiert, andererseits wird sich mit den strukturellen Hintergründen von Flucht und Migration und somit der Forderung nach gerechter bzw. selbstbestimmter Entwicklung beschäftigt (AEI 2014).

In Mali ist eine der größten Gruppen von AEI aktiv, die kleinbäuerliche Basisgewerkschaft COPON mit mehreren hundert Mitgliedern. Sie hat sich in der malischen Sonderverwaltungszone Office du Niger gegründet. Nach dem Ersten Weltkrieg hatte die französische Kolonialadministration die Idee, den Niger zur Bewässerung eines riesigen Gebiets in der Sahelzone zu nutzen (Coulibaly 2015). Baumwollanbau sollte die heimische Textilindustrie sanieren. Nach der Gründung der gleichnamigen Verwaltungsbehörde „Office du Niger“ in den 1930er Jahren wurden Bäuerinnen und Bauern dorthin zwangsumgesiedelt. Die kolonialen Abhängigkeitsverhältnisse zwischen Frankreich und den zur Arbeit gezwungenen Bewohner*innen von Französisch-Sudan finden heute ihre Fortführung in der Beziehung zwischen malischen Verwaltungs- und Wirtschaftseliten, die bestehende kleinbäuerliche Landwirtschaft als rückständig diskreditieren, und der Landbevölkerung in der Ségou Region (Coulibaly 2015).

Die Verwaltungs- und Rechtsstruktur des Office du Niger wird von Aktivist*innen gegen Landgrabbing in Mali als eine Fortführung der kolonialen Landnahme und Zwangsstruktur beschrieben: „Das war ein Komplott des weißen Kolonisators gegen die kolonisierte Bevölkerung. Und es war ein Komplott des schwarzen Kolonisators, der die gleiche Grund- und Bodenordnung des weißen Kolonisators genommen hat und diese gegen seine eigene Bevölkerung angewendet hat" (Interview mit Aktivist*in und NGO-Mitarbeiter*in am 25.10.2018 in Bamako, Mali). Durch eine Allianz zwischen „bürokratischer Bourgeoisie - also dem Staatsapparat - und der Wirtschaftsbourgeoisie“ aus dem In- und Ausland würden „Bauern 
enteignet und unsere Landwirtschaft für den internationalen Markt freigegeben“ (Interview mit Aktivist*in am 19.10.2019 in Bamako, Mali).

Es hat aber immer, auch zur Kolonialzeit, kleinbäuerlichen Widerstand gegeben (Coulibaly 2015). Seit der Jahrtausendwende hat es zwischen 2005 und 2008 schon einen Zyklus des Widerstandes gegen Landgrabbing durch die Administration des Office du Niger gegeben, der aber mittels Repression erstickt wurde (Bernau 2012). Die COPON gründete sich als Reaktion auf ein bundesdeutsches Entwicklungsprojekt. Die KfW hatte 2013 und 2014 im Rahmen des Projekts „Siengo Extension“ knapp 1.500 Hektar Ackerland für den Reisanbau erschlossen. Doch ein großer Teil der Flächen wurde von den verantwortlichen Beamt*innen Malis nicht an die hierfür vorgesehenen Familien verteilt, sondern an vermögende Dritte weiterverkauft. Jegliche Verantwortung dafür wies die deutsche Regierung in Gesprächen mit und Briefen an AEI von sich.

Deswegen hat AEI zu diesem Fall transnational sowohl in Mali als auch in Deutschland Öffentlichkeitsarbeit gemacht und Protestaktionen organisiert. Das hat unter anderem dazu geführt, dass der Fall nochmals von der KfW untersucht wurde und die malische Administration die Kleinbäuerinnen und -bauern deutlich weniger repressiv behandelt. Der transnationale Druck in Mali sowie auf Entwicklungshilfegeber wie dem BMZ hat nach Aussage von COPON-Mitgliedern dazu geführt, dass die Behörden vorsichtiger und auch nachsichtiger geworden seien, was die Wegnahme von gepachtetem Land angeht (Interviews mit Mitgliedern der COPON im Februar 2018). Somit würden die Aktivitäten von AEI eine Prävention gegen Landgrabbing darstellen. Darüber hinaus hat die Gewerkschaft es mittlerweile erreicht, dass ihr für ihre Mitglieder 200 Hektar Ackerfläche zugesprochen wurden (Interviews mit Mitgliedern der COPON und Aktivist*innen von Afrique-Europe-Interact im Oktober 2018). Nur muss sie selbst für die Erschließung sorgen, was finanziell ein Ding der Unmöglichkeit ist. Deswegen versucht AEI nun, die deutsche Bundesregierung dazu zu bringen, die Kosten dafür zu übernehmen. Die transnationale Praxis von Afrique-EuropeInteract verweist auf eine dekoloniale Zukunft, in der kleinbäuerliche Abhängigkeit von ausländischen und nationalen Eliten verringert und Autonomie gestärkt wird.

\section{Ausblick}

Es sollte bei entwicklungspolitischen Anstrengungen, wenn sie sich - wie auch für den entwicklungspolitischen Freiwilligendienst Weltwärts der Fall - globaler Gleichheit und Gerechtigkeit und umfassender Dekolonisierung verpflichtet sehen, entsprechend um eine Kritik an „asymmetrischer Globalisierung, ungleichen Machtverhältnissen, und Eliten aus Nord und Süd, die ihre eigenen Annahmen [den Marginalisierten dieser Welt] als universell aufzwängen“ (Andreotti 2006: 47) gehen. Ausgehend von einer auch die Prinzipien der europäischen Aufklärung ernstnehmenden Gleichberechtigung aller Menschen müssten die in der BRD 
vorherrschenden Extraktions-, Produktions- und Konsummuster - die nur einer exklusiven Minderheit dienen, Ausbeutung von Menschen sowie Zerstörung von Natur bedeuten und auf der Fortführung kolonialer Strukturen gründen - beendet werden, anstatt eine wachsende Bevölkerung im Süden für globale Probleme verantwortlich zu machen. Aller Nachhaltigkeitsrhetorik zum Trotz wird allerdings in der deutschen Entwicklungspolitik im In- und Ausland weiterhin nicht die in der BRD vorherrschende imperiale Lebensweise, die auf der Fortführung kolonialer Ausbeutung beruht, als zentrales „Entwicklungsproblem“ in den Blick genommen.

Darüber hinaus ist in Bezug auf den Umgang mit dem kolonialen Erbe - neben dem Recht auf globale Bewegungsfreiheit - die Frage von kulturellen und materiellen Reparationen anzugehen. Entwicklungshilfe wird zwar vor allem von Seiten der „Geber“ bisweilen als eine solche Kompensation interpretiert, aber zum einen ist sie in vielen Fällen nicht am Wohl des Großteils der Gesellschaften im Süden orientiert, und zum anderen erfolgt sie mit dem paternalistischen Gestus der Großzügigkeit und nicht als Wiedergutmachung für begangene Verbrechen. Und wie im Fall des kleinbäuerlichen Widerstands in Mali müsste sie sich auch dem Problem stellen, dass sie innergesellschaftliche Ungleichheit stärken kann. Für notwendig erachte ich - auch für die Zukunft des Weltwärts-Programms - transnationale mit innergesellschaftlicher Solidarität zu verbinden: mit von Hartz IV und dem Migrations- und Asylsystem Betroffenen, aber auch beispielsweise mit Bauern und Bäuerinnen in Brandenburg, die ihr Land an Großinvestoren verlieren, oder Mieter*innen, die aus den deutschen Innenstädten verdrängt werden.

\section{Literaturverzeichnis}

Afrique-Europe-Interact (2014): „Kurzpräsentation unseres Netzwerks“, https://afriqueeurope-interact.net/38-0-Unser-Netzwerk.html, 30.01.2019.

Andreotti, Vanessa (2006): Soft versus Critical Citizenship Education, in: Policy \& Practice: A Development Education Review 3, S. 40-51.

Angenendt, Steffen und Silvia Popp (2014): Bevölkerungswachstum, Fertilität und Kinderwunsch. Herausforderungen für die Entwicklungszusammenarbeit am Beispiel Subsahara-Afrikas, in: SWP-Studien, Heft 20.

Bayer HealthCare (2011): Wir kooperieren mit USAID, um einen nachhaltigen Markt für Verhütungsmittel in Afrika zu schaffen - Bayer HealthCare Pharmaceuticals, www.bayerhealthcarepharmaceuticals.com/de/presse/im_fokus/contraceptive_security_initiative. php, 20.11.2015.

Bechtum, Alexandra und Bernd Overwien (2017): Kann postkoloniale Kritik Schule machen? Über ihre Grenzen und Potenziale für (entwicklungs-)politische Bildungsarbeit, in: Entwicklungstheorie von heute - Entwicklungspolitik von morgen, hrsg. von Hans-Jürgen Burchardt, Stefan Peters und Nico Weinmann, Baden-Baden, S. 59-84, https://doi. org/10.5771/9783845267340-60. 
Bendix, Daniel (2016): The colonial present in international development: The case of German interventions in obstetric care in Tanzania, in: Progress in Development Studies, Jg. 16., Heft 3, S. 229-243.

Bendix, Daniel und Susanne Schultz (2017): The Political Economy of Family Planning: Population Dynamics and Contraceptive Markets, in: Development and Change, Jg. 49, Heft 2, S. 259-85.

Bernau, Olaf (2012): Komplexe Realitäten. Kleinbäuerliche Landwirtschaft steht in Mali unter massivem Druck, in: analyse \& kritik. Zeitung für linke Debatte und Praxis, Heft 574, https://www.akweb.de/ak_s/ak574/17.htm, 30.01.2019.

Bernau, Olaf (2017): Anlageobjekt Afrika. Die deutsche G20-Präsidentschaft propagiert »Investitionspartnerschaften « im Kampf gegen Armut und Hunger, in: analyse \& kritik. Zeitung für linke Debatte und Praxis, Heft 628, S. 14.

BMZ - Bundesministerium für wirtschaftliche Zusammenarbeit und Entwicklung (2013): Bevölkerungsdynamik in der deutschen Entwicklungszusammenarbeit - Positionspapier, 10. BMZ-Strategiepapier, Bonn-Berlin.

BMZ - Bundesministerium für wirtschaftliche Zusammenarbeit und Entwicklung (2019a): Programm. Der entwicklungspolitische Freiwilligendienst stellt sich vor, www.weltwaerts.de/de/programm.html, 30.01.2019.

BMZ - Bundesministerium für wirtschaftliche Zusammenarbeit und Entwicklung (2019b): Über weltwärts. weltwärts - Der entwicklungspolitische Freiwilligendienst stellt sich vor, www.weltwaerts.de/de/ueber-weltwaerts.html, 30.01.2019.

Brämer, Josephine und Aram Ziai (2015): Die deutsche Entwicklungspolitik unter Niebel: Eine handlungslogische Analyse des FDP-geführten BMZ, in: Peripherie - Politik. Ökonomie. Kultur, Jg. 35, Heft 140, S. 400-418, https://doi.org/10.3224/peripherie.v35i140.22996.

Brand, Ulrich und Markus Wissen (2017): Imperiale Lebensweise. Zur Ausbeutung von Mensch und Natur im globalen Kapitalismus, München.

Brot für die Welt (2013a): Lebensmittelspekulation: Ein mörderisches Spiel, http://weyandt. de/wp-content/uploads/2013/12/plakat_banane-300x212.jpg, 30.04.2017.

Brot für die Welt (2013b): Verspielen Sie nicht das Leben von Menschen, www.billyboard.de/ verspielen-sie-nicht-das-leben-von-menschen/leben-verspielen/, 30.04.2017.

BUKO Pharma-Kampagne (2014): Arm und vergessen. Untersuchung des Geschäftsverhaltens von Boehringer Ingelheim, Bayer und Baxter in Uganda, Pharma-Brief Spezial, Nr. 1.

Coulibaly, Chéibane (2015): Politiques agricoles et stratégies paysannes au Mali de 1910 à 2010. Mythes et réalités à l'Office du Niger, Bamako.

Danielzik, Chandra-Milena (2013): Überlegenheitsdenken fällt nicht vom Himmel. Postkoloniale Perspektiven auf Globales Lernen und Bildung für nachhaltige Entwicklung, in: ZEP, Jg. 13, Heft 1, S. 26-33.

decolonize orientierungsrahmen! (2014): decolonize orientierungsrahmen!, https://decolonizeorientierungsrahmen.wordpress.com, 30.04.2017.

Deutsche Gesellschaft für Eingebornenschutz (1914): Eingebornenschutz, in: Koloniale Rundschau - Monatsschrift für die Interessen unserer Schutzgebiete und ihrer Bewohner, Nr. 1, S. 1-5.

Deutsche Welle (2012): Seven Billion and Climbing, but not Everywhere, http://dw.com/ $\mathrm{p} / 15 \mathrm{~V} 5 \mathrm{~W}, 30.04 .2017$. 
Deutsches Reichsgesetzblatt (1885): Generalakte der Berliner Konferenz. Nr. 23, Berlin.

Escobar, Arturo (1994): Encountering Development. The Making and Unmaking of the Third World, Princeton.

FIAN (2017): Coffee to Go - Landvertreibung zugunsten der Kaweri Coffee Plantation in Uganda. Eine menschenrechtliche Analyse, www.fian.de/fileadmin/user_upload/dokumente/shop/Fallarbeit/FS_Coffee_To_Go_2017_1.pdf, 30.01.2019.

Friedrichsmeyer, Sara, Sara Lennox und Susanne Zantop (Hrsg.) (1998): The Imperialist Imagination: German Colonialism and its Legacy, Michigan.

Haas, Benjamin (2012): Ambivalenz der Gegenseitigkeit: Reziprozitätsformen des weltwärtsFreiwilligendienstes im Spiegel der Postkolonialen Theorie, Köln.

Hall, Stuart (1996): When was "the post-colonial"? Thinking at the limit, in: The Post-Colonial Question. Common Skies, Divided Horizons, hrsg. von Iain Chambers und Lidia Curti, London, S. 242-60.

Kiesel, Timo und Daniel Bendix (2010): White Charity: Eine postkoloniale, rassismuskritische Analyse der entwicklungspolitischen Plakatwerbung in Deutschland, in: Peripherie, Jg. 30, Heft 120, S. 482-95.

KMK und BMZ (Hrsg) (2007): Orientierungsrahmen für den Lernbereich Globale Entwicklung im Rahmen einer Bildung für nachhaltige Entwicklung, Bonn-Berlin.

KMK und BMZ (2014): Orientierungsrahmen für den Lernbereich Globale Entwicklung im Rahmen einer Bildung für nachhaltige Entwicklung (Arbeitsdokument - zur Anhörung und Kommentierung freigegeben), 2. Aufl., Berlin.

KMK und BMZ (2015): Orientierungsrahmen für den Lernbereich Globale Entwicklung im Rahmen einer Bildung für nachhaltige Entwicklung, 2. Aufl., Berlin.

Kontzi, Kristina (2015): Postkoloniale Perspektiven auf „weltwärts“ Ein Freiwilligendienst in weltbürgerlicher Absicht, Baden-Baden.

Lincoln, Sarah (2013): Wirtschaft versus Menschenrechte - Niebel versucht Vorwürfe gegen deutschen Kaffeehersteller zu unterbinden, 20.08.2013, https://info.brot-fuer-die-welt.de/ blog/wirtschaft-versus-menschenrechte-niebel-versucht, 30.01.2019.

Misereor (2011): Mut ist, www.kolle-rebbe.de/arbeiten/mut-ist/, 30.04.2017.

Transparency Market Research (2014): Contraceptives Market - Global Industry Analysis, Size, Share and Forecast 2014-2020, www.transparencymarketresearch.com/contraceptivesmarket.html, 30.04.2017. 